\title{
FATOU'S LEMMA AND LEBESGUE'S CONVERGENCE THEOREM FOR MEASURES ${ }^{1}$
}

\author{
ONÉSIMO HERNÁNDEZ-LERMA ${ }^{2}$ \\ CINVESTA V-IPN, Departamento de Matemáticas \\ Apdo. Postal 14-740, México D.F. 07000, Mexico \\ E-mail: ohernand@math.cinvestav.mx \\ JEAN B. LASSERRE \\ $L A A S-C N R S$ \\ 7, Avenue Du Colonel Roche \\ 31077 Toulouse Cédex, France \\ E-mail: lasserre@laas.fr
}

(Received December, 1998; Revised November, 1999)

\begin{abstract}
Analogues of Fatou's Lemma and Lebesgue's convergence theorems are established for $\int f d \mu_{n}$ when $\left\{\mu_{n}\right\}$ is a sequence of measures. A "generalized" Dominated Convergence Theorem is also proved for the asymptotic behavior of $\int f_{n} d \mu_{n}$ and the latter is shown to be a special case of a more general result established in vector lattices and related tot he DunfordPettis property in Banach spaces.
\end{abstract}

Key words: Measures, Fatou and Lebesgue's Theorems, AM-Spaces, AL-Spaces.

AMS subject classifications: $28 \mathrm{~A} 33,28 \mathrm{C} 15$.

\section{Introduction}

One purpose of this paper is to derive analogues of Fatou's Lemma and of the Monotone and the dominated Convergence Theorems for measures instead of functions. We show that these theorems with respect to a sequence of measurable functions $\left\{f_{n}\right\}$ and some measure $\mu$ also hold for a sequence of measures $\left\{\mu_{n}\right\}$ and a function $f$. In the Monotone and Dominated Convergence Theorems, one formally replaces the point

${ }^{1}$ Research partially supported by the CNRS (France)-CONACYT (México) Scientific Cooperation Program, and the ECOS-ANUIES (France-México) Cooperation Program as well.

${ }^{2}$ The research of the first author was also partially supported by a visiting appointment at LAAS-CNRS, Toulouse, France by CONACTYT Grant 3115PE9608. 
wise convergence of $f_{n}$ to $f$ by its natural counterpart for set functions, namely, the setwise convergence of $\mu_{n}$ to $\mu$.

As in Langen [8], Serfozo [12], and Hernández-Lerma and Lasserre [3], we also consider the asymptotic properties of $\int f_{n} d \mu_{n}$ for sequences of functions $f_{n}$ and measures $\mu_{n}$ and provide "generalized" versions of Fatou's Lemma and Lebesgue's Dominated Convergence Theorem. We show that coupling the assumptions of both Dominated Convergence Theorems for functions and for measures yields a natural "generalized" Dominated Convergence Theorem.

Another purpose of this paper is to show that the latter can be obtained as a special case of a more general result which we establish for the convergence of a sequence $\left\langle y_{n}, x_{n}\right\rangle$ in a dual pair of vector lattices. This result is a variant of the DunfordPettis property in Banach spaces.

In Section 2 we present the main results for asymptotic properties of $\int f d \mu_{n}$ and $\int f_{n} d \mu_{n}$. Some examples of applications are also briefly discussed.

In Section 3, we present a "generalized" Dominated Convergence Theorem for the convergence of $\left\langle x_{n}, y_{n}\right\rangle$ for two sequences $\left\{x_{n}\right\}$ and $\left\{y_{n}\right\}$ in appropriate spaces and relate the result to the Dunford-Pettis property.

\section{Convergence Theorem in Measurable Spaces}

In this section, we consider a measurable space $(X, \mathfrak{B})$, a sequence of measures $\left\{\mu_{n}\right\}$ on $\mathfrak{B}$, and a real-valued measurable nonnegative function $f$ on $X$.

\subsection{Fatou's Lemma and Lebesgue's Convergence Theorems for Measures}

We have the following result:

Theorem 2.1: Let $f$ be a real-valued nonnegative measurable function on $X$, and let $\left\{\mu_{n}\right\}$ be a sequence of measures on $\mathfrak{B}$.

(a) "Fatou". If $\liminf _{n \rightarrow \infty} \mu_{n}(A) \geq \mu(A) \forall A \in \mathfrak{B}$ for some measure $\mu$, then

$$
\liminf _{n \rightarrow \infty} \int f d \mu_{n} \geq \int f d \mu .
$$

(b) "Dominated". If $\mu_{n}(A) \rightarrow \mu(A) \forall A \in \mathfrak{B}$ and $\mu_{n} \leq \nu$ for some measures $\mu$ and $\nu$ such that $\int f d \nu<\infty$, then

$$
\lim _{n \rightarrow \infty} \int f d \mu_{n}=\int f d \mu .
$$

(c) "Monotone". If $\mu_{n} \leq \mu_{n+1}$ for all $n$, there is a measure $\mu$ such that $\mu_{n}(A) \rightarrow \mu(A) \forall A \in \mathscr{B}$ and

$$
\lim _{n \rightarrow \infty} \int f d \mu_{n}=\int f d \mu .
$$

Proof: (a) Every nonnegative measurable function $f$ is the pointwise limit of a nondecreasing monotone sequence $\left\{f_{m}\right\}$ of simple functions, i.e., $f_{m} \uparrow f$ and $f_{m}=$ $\sum_{k=1}^{p_{m} \lambda_{m k} 1_{B} B_{m k}}$ where $\lambda_{m k} \geq 0$ and $B_{m k} \in \mathscr{B}, k=1, \ldots, p_{m}$. Therefore, for every $m=1,2, \ldots$, we have: 


$$
\begin{gathered}
\liminf _{n \rightarrow \infty} \int f d \mu_{n} \geq \liminf _{n \rightarrow \infty} \int f_{m} d \mu_{n} \\
=\liminf _{n \rightarrow \infty} \sum_{k=1}^{p_{m}} \lambda_{m k} \mu_{n}\left(B_{m k}\right) \\
\geq \int f_{m} d \mu .
\end{gathered}
$$

Hence, as $f_{m} \uparrow f$, by the standard Monotone Convergence Theorem for functions (see for instance, $[1,11])$, we get

$$
\liminf _{n \rightarrow \infty} \int f d \mu_{n} \geq \int f d \mu
$$

which is $(2.1)$. (Notice that $f$ need not be $\mu$-integrable.)

To get $(b)$, apply $(a)$ to the sequences $\left\{\nu-\mu_{n}\right\}$ and $\left\{\mu_{n}\right\}$ so that

$$
\limsup _{n \rightarrow \infty} \int f d \mu_{n} \leq \int f d \mu \leq \liminf _{n \rightarrow \infty} \int f d \mu_{n}
$$

which implies $(b)$.

To get $(c)$, note that as $\left\{\mu_{n}\right\}$ is increasing, there is a measure $\mu$ such that $\mu_{n}(A) \uparrow \mu(A)$ for every $A \in \mathfrak{B}$ (see Chapter III in Doob [1]). From (a)

$$
\int f d \mu \leq \liminf _{n \rightarrow \infty} \int f d \mu_{n}
$$

Now, as $f$ is nonnegative and $\mu_{n} \uparrow \mu$,

$$
\liminf _{n \rightarrow \infty} \int f d \mu_{n}=\lim _{n \rightarrow \infty} \int f d \mu_{n} \leq \int f d \mu,
$$

which is combined with (2.4) yields (2.3).

Theorem 2.1 $(a)$ is an analogue for measures of the celebrated Fatou's Lemma for functions, whereas $(b)$ (resp. $(c)$ ) is the analogue for measures of the Lebesgue's Dominated (resp. Monotone) Convergence Theorem for functions.

Examples of minorizing measures $\mu$ in (2.1) are:

- The order-liminf of $\mu_{n}$ defined as $O$ - $\liminf _{n \rightarrow \infty} \mu_{n}:=\vee_{n \geq 1} \wedge_{m \geq n} \mu_{m}$ (e.g. see [6] or Chapter XII of [14]).

- $\mu:=$ the lower envelope of $\left\{\mu_{n}\right\}$, already used in [7].

Hence, for instance, using the order-lim inf we may rewrite (2.1) as

$$
\liminf _{n \rightarrow \infty} \int f d \mu_{n} \geq \int f d\left(\vee_{n \geq 1} \wedge_{m \geq n} \mu_{m}\right) \text {. }
$$

\subsection{Asymptotic Properties of $\int f_{n} d \mu_{n}$}

Let us now investigate the asymptotic properties of $\int f_{n} d \mu_{n}$ for two sequences $\left\{f_{n}\right\}$ and $\left\{\mu_{n}\right\}$ of functions and measures, respectively. The next theorem shows "generalized versions of Theorem 2.1(a), (b).

Theorem 2.2: Let $\left\{\mu_{n}\right\}$ and $\left\{f_{n}\right\}$ be two sequences of measures and measurable functions respectively. 
(i) "Generalized Fatou". Let $\mu$ be a measure such that $\mu(A) \geq \liminf _{n} \mu_{n}(A)$ for every $A \in \mathfrak{B}$, and let $f(x):=\liminf _{n} f_{n}(x), x \in X$. If $\bar{f}_{n} \geq g$ for all $n$ with $g$ measurable and $\lim _{n} \int g d \mu_{n}=\int g d \mu>-\infty$, then

$$
\liminf _{n \rightarrow \infty} \int f_{n} d \mu_{n} \geq \int f d \mu \text {. }
$$

(ii) “Generalized Dominated". Assume that:

(a1) $\left|f_{n}\right| \leq g \forall n$ for some measurable function $g \geq 0$.

(a2) $\mu_{n} \leq \nu \forall n$ for some measure $\nu$.

(b1) $\quad f_{n}(\bar{x}) \rightarrow f(x)$ a.e. $[\nu]$.

(b2) $\mu_{n}(A) \rightarrow \mu(A) \forall A \in \mathscr{B}$ for some measure $\mu$.

(c) $\quad \int g d \nu<\infty$.

Then

$$
\lim _{n \rightarrow \infty} \int f_{n} d \mu_{n}=\int f d \mu .
$$

Proof: $(i)$ We first prove (2.6) for a nonnegative sequence $\left\{f_{n}\right\}$, and then the general case follows by applying the result to the nonnegative sequence $\left\{f_{n}-g\right\}$. Therefore, assume that $f_{n} \geq 0 \forall n$. For every (fixed) arbitrary $n_{0}$ we have:

$$
\int f_{n} d \mu_{n} \geq \int\left[\inf _{m \geq n_{0}} f_{m}\right] d \mu_{n}, \quad \forall n \geq n_{0} .
$$

Therefore, from Theorem 2.1(a),

$$
\liminf _{n \rightarrow \infty} \int f_{n} d \mu_{n} \geq \int\left[\inf _{m \geq n_{0}} f_{m}\right] d \mu .
$$

But we also have $\left[\inf _{m} \geq n_{0} f_{m}\right] \uparrow f:=\liminf _{n} f_{n}$ as $n_{0} \rightarrow \infty$. Therefore, by the standard Monotone Convergence Theorem,

$$
\liminf _{n} \int f_{n} d \mu_{n} \geq \lim _{n \rightarrow \infty} \int\left[\inf _{m \geq n} f_{m}\right] d \mu=\int f d \mu
$$

which is the desired result (2.6).

(ii) In view of $(a 2),(b 2)$ and (c), from Theorem $2.1(b)$ one has $\int g d \mu_{n} \rightarrow \int g d \mu$. Also, observe that $f$ is measurable as it is the pointwise limit of measurable functions.

As $g \pm f_{n} \geq 0 \forall n$, from (2.6) we get $\liminf _{n} \int\left(g \pm f_{n}\right) d \mu_{n} \geq \int \liminf _{n}\left(g \pm f_{n}\right) d \mu$. Thus, since $\int g d \mu \leq \int g d \nu<\infty$, and noting that from $\nu \geq \mu$, the $\nu$-a.e. convergence $f_{n} \rightarrow f$ implies its $\mu$-a.e. convergence

$$
\limsup _{n \rightarrow \infty} \int f_{n} d \mu_{n} \leq \int f d \mu \leq \liminf _{n \rightarrow \infty} \int f_{n} d \mu_{n}
$$

which yields the desired result (2.7).

Theorem 2.2( $i$ ) has also been proved by Serfozo (see Lemma 2.2. in [12]) with the same hypotheses we use and $f_{n}$ nonnegative but with a longer proof, and also in Royden (see Chapter 11, Proposition 17 in [11]) under stronger hypotheses (namely, $f_{n} \rightarrow f$ pointwise and $\mu_{n} \rightarrow \mu$ setwise).

Theorem 2.2(ii), on the other hand, has also been proved in Royden (Chapter 11, Proposition 18, in [11]) and Serfozo [12] under different hypotheses. In [11], one assumes $(a 1),\left|f_{n}\right| \leq g_{n}$ with $\lim _{n} g_{n}(x)=g(x) \forall x$, and $\lim _{n} \int g_{n} d \mu_{n}=\int g d \mu<\infty$, whereas in [12], one requires $(a 1), \liminf _{n} \mu_{n}(A) \geq \mu(A) \forall A \in \mathscr{B}$ and $\left|f_{n}\right| \leq g_{n} \forall n$ 
with $\limsup \sup _{n} \int g_{n} d \mu_{n}=\int\left(\liminf _{n} g_{n}\right) d \mu<\infty$. These assumptions are weaker than those of Theorem 2.2, but the assumption that there exists a sequence $g_{n}$ such that $\int g_{n} d \mu_{n} \rightarrow \int g d \mu$ is not easy to check, and in fact, this assumption is similar to the result one wants to prove.

We believe Theorem 2.2(ii) is more natural and more in the spirit of the "traditional" Dominated Convergence Theorem.

Indeed, Lebesgue's Dominated Convergence Theorem states that if $\left\{f_{n}\right\}$ is a sequence of measurable functions on $X$, with $\left|f_{n}\right| \leq g \forall n$, ( $g$ integrable) and such that $f_{n} \rightarrow f$ pointwise, then $\int f_{n} d \mu \rightarrow \int f d \mu$.

Hence, $(a 1),(b 1)$ (resp. $(a 2),(b 2))$ are the assumptions in the Dominated Convergence Theorem for functions (resp. for measures) whereas $(c)$ links $(a 1)$ and $(a 2)$. Combination of both yields the generalized Dominated Convergence Theorem 2.2(ii).

\subsection{Weak Convergence}

A frequent case encountered in practice is when $\left\{\mu_{n}\right\}$ is a sequence of finite measures on $(X, \mathfrak{B})$ and instead of the setwise convergence of $\mu_{n}$ to $\mu$ (which in general is hard to establish), one often has a much weaker type of convergence, for instance

$$
\int g d \mu_{n} \rightarrow \int g d \mu \forall g \in G
$$

where $G$ is a set of bounded measurable functions that separate points in $M(X)$, and $M(X)$ is the Banach space of finite signed measures on $(X, \mathscr{B})$, equipped with the total variation norm. The convergence in (2.8) is the convergence in the weak topology on $M(X)$ generated by $G$, denoted $\sigma(M(X), G)$. For instance, for a sequence $\left\{\mu_{n}\right\}$ of probability measures on a locally compact Hausdorff space $X$ (with $\mathfrak{B}$ its usual Borel $\sigma$-field), typical choices for $G$ are:

(i) $\quad G=C_{0}(X)$, the space of continuous functions that vanish at infinity;

(ii) $\quad G=C_{b}(X)$, the space of bounded continuous functions on $X$.

When (2.8) holds with $G$ as in (i) (resp. (ii)), one has the usual "vague" (resp. "weak") convergence of probability measures in the weak topology $\sigma\left(M(X), C_{0}(X)\right)$ (resp. weak topology $\left.\sigma\left(M(X), C_{b}(X)\right)\right)$. One then has the following Dominated Convergence Theorem.

Proposition 2.3: Let $f$ be a nonnegative measurable function and let $\left\{\mu_{n}\right\}$ and $\mu$ be such that $\int g d \mu_{n} \rightarrow \int g d \mu$ for every $g \in G$, where $G$ is a set of bounded measurable functions that separates points in $M(X)$. If $\mu_{n} \leq \nu \forall n$ for some finite measure $\nu$, then, as $n \rightarrow \infty$,

(a) $\quad \mu_{n}(A) \rightarrow \mu(A) \forall A \in \mathscr{B}$.

In addition, if $\int f d \nu<\infty$, then

(b) $\int f d \mu_{n} \rightarrow \int f d \mu$.

Proof: As $\nu$ is finite and $\mu_{n} \leq \nu$, the sequence $\left\{\mu_{n}\right\}$ is norm-bounded (that is, bounded in the norm of $M(X))$ and the countable additivity of the $\mu_{n}$ 's is uniform in $n$. Therefore, $\left\{\mu_{n}\right\}$ is sequentially compact in the weak topology $\sigma\left(M(X), M(X)^{*}\right)$, (see Theorem IV.9.1 in [2]). In particular, every accumulation point $\varphi \in M(X)$ of a converging subsequence $\left\{\mu_{n_{k}}\right\}$ satisfies $\mu_{n_{k}}(A) \rightarrow \varphi(A) \forall A \in \mathfrak{B}$. Moreover, we also have $\int g d \mu_{n} \rightarrow \int g d \mu$ for every $g \in G$. Hence, identifying $G$ with a subset of $M(X)^{*}$ and using the fact that $G$ separates points in $M(X)$, we must have $\varphi=\mu$. As $\varphi$ was an arbitrary accumulation point, we get $(a)$. 
(b) follows from Theorem 2.1(b).

As an application in Stochastic Control Theory, consider the sequence of probability measures $\mu_{n}:=Q\left(\cdot \mid x, a_{n}\right)$ on $(X, \mathscr{B})$ with $X$ being a locally compact separable metric space and $Q$ a stochastic kernel. Then, $\mu_{n} \leq \nu \forall n$ implies, e.g., "tightness" of the sequence $\left\{\mu_{n}\right\}$ which in turn is used to prove the existence of optimal controls (see [4, 7]). Similarly, if $\mu_{n}:=P^{n}(\cdot \mid x)$ denotes the sequence of $n$ step transition kernels (with initial state $x \in X$ ) of a stochastic (Markov) kernel $P(\cdot \mid x)$, and if $\mu_{n} \leq \nu$ for some finite measure $\nu$, then we obtain the "constrictiveness" of the stochastic kernel $P$, which yields the "Spectral Decomposition Theorem" (see [9]).

Another typical application is, for instance, to study the ergodic behavior of functionals of Markov chains. Consider a Markov chain $\left\{x_{t}, t=0,1, \ldots\right\}$ on a locally compact separable metric space $X$ and suppose one wants to evaluate the limit (if it exists) when $n \rightarrow \infty$ of $j_{n}(\omega):=n^{-1} \sum_{t=0}^{n-1} f\left(X_{t}(\omega)\right)$ on a sample-path $\left\{X_{t}(\omega)\right\}$ and for some nonnegative (possibly) unbounded (and possibly not continuous) function $f$. Write $j_{n}(\omega)=\int f d \mu_{n}^{\omega}$ where $\mu_{n}^{\omega}$ is the $n$-step occupation (probability) measure for the sample path $\omega$. It has been shown (e.g., see [10]) that $\mu_{n}^{\omega} \rightarrow \mu P_{x^{-a . s .}}$ in the vague topology and $\mu$ is a (possibly trivial) invariant measure for the Markov chain $\left\{X_{t}\right\}$.

Thus, if for some finite measure $\nu, \mu_{n}^{\omega} \leq \nu \forall n$ and $\int f d \nu<\infty$, then the direct application of Proposition 2.3 yields

$$
\lim _{n \rightarrow \infty} j_{n}(\omega)=\int f d \mu P_{x^{- \text {a.s. }}}
$$

with $\mu$ being an invariant probability measure.

\subsection{Locally Compact Spaces}

When the underlying space $X$ is a locally compact separable metric space, specialized results are known for Fatou's and Dominated Convergence theorems using weak convergence of measures and continuous convergence of functions (see Langen [8], Serfozo [12] and references therein).

However, we would like to point out part $(b)$ in the proposition below $[(a)$ is already well known for a sequence of "bounded" measures $\mu_{n}$ ] that might be useful when dealing with a sequence of possibly infinite measures that converge in a weak sense to be precised below.

Let $X$ be a locally compact separable metric space with $\mathscr{B}_{B}$ as its usual Borel $\sigma$ field, and let $C_{0}(X)$ be the (separable) Banach space of real-valued continuous functions that vanish at infinity.

Proposition 2.4: Let $f$ be a nonnegative lower-semicontinuous function on $X$ and let $\left\{\mu_{n}\right\}$ be a sequence of measures such that

$$
\int h d \mu_{n} \rightarrow \int h d \mu \forall h \in C_{0}(X)
$$

for some measure $\mu$. Then:

(a) $\quad \liminf _{n \rightarrow \infty} \int f d \mu_{n} \geq \int f d \mu$.

(b) If, in addition, $\mu_{n} \leq \nu$ for some measure $\nu$ such that $\int f d \nu<\infty$, then

$$
\lim _{n \rightarrow \infty} \int f d \mu_{n}=\int f d \mu .
$$


Proof: (a) As $f$ is nonnegative and lower semi-continuous, there exists an increasing sequence of nonnegative continuous bounded functions $v_{k}$ on $X$ such that $v_{k}(x) \uparrow$ $f(x) \forall x \in X$. Similarly, as each $v_{k}$ is a nonnegative continuous bounded function and $X$ is $\sigma$-compact $([11$, p. 203, Theorem 21]), for every $k$, there is an increasing sequence $\left\{v_{k l}, l=1,2, \ldots\right\}$ of nonnegative functions $v_{k l}$ in $C_{0}(X)$ with $v_{k l}(x) \uparrow v_{k}(x)$ for all $x \in X$ as $l \rightarrow \infty$. Hence

$$
\begin{gathered}
\liminf _{n} \int f d \mu_{n} \geq \liminf _{n} \int v_{k} d \mu_{n} \quad \forall k \\
\geq \liminf _{n} \int v_{k l} d \mu_{n} \quad \forall k, l \\
=\int v_{k l} d \mu[\text { by (2.9)]. }
\end{gathered}
$$

Thus, letting $l \rightarrow \infty$ and then $k \rightarrow \infty$ the result $(a)$ follows.

(b) Apply (a) to the nonnegative sequences $\left\{\nu \pm \mu_{n}\right\}$, which immediately yields

$$
\limsup _{n \rightarrow \infty} \int f d \mu_{n} \leq \int f d \mu \leq \liminf _{n \rightarrow \infty} \int f d \mu_{n}
$$

as the desired result.

In the general setting, a result like (2.10) requires the setwise convergence of $\mu_{n}$ to $\mu$, which is implied by the condition $\mu_{n} \leq \nu$ when $\nu$ is finite.

The important thing in Proposition 2.4 is that (2.9) and the condition $\mu_{n} \leq \nu \forall n$ do not imply that $\mu_{n} \rightarrow \mu$ setwise if $\nu$ is not finite.

\section{A "Generalized" Dominated Convergence}

We recall that a Banach lattice $\mathscr{S}$ with a positive cone $S$ is called an $A M$-space if. the norm has the following property

$$
\|x \vee y\|=\|x\| \vee\|y\|, x, y \in S
$$

A typical example on an $A M$-space is $B(X)$, the Banach space of bounded measurable functions on the measurable space $(X, \mathfrak{B})$, with the sup-norm.

On the other hand, a Banach lattice $\mathscr{S}$ with positive cone $S$ is an $A L$-space (or abstract Lebesgue space) if

$$
\|x+y\|=\|x\|+\|y\|, x, y \in S .
$$

A typical example of an $A L$-space is $\mathscr{G}:=L_{1}(X, \mathfrak{B}, \mu)$ for some measure space $(X, \mathfrak{B}, \mu)$ or the space of $\mathscr{G}:=M(X)$, the Banach space of finite signed measures on $\mathscr{B}$, for some measurable space $(X, \mathscr{B})$, and the total variation norm.

Every $A M$-space or $A L$-space $\mathscr{S}$ has the so-called Dunford-Pettis property, that is, for a sequence $\left\{x_{n}\right\}$ in $\mathscr{S}$, a sequence $\left\{y_{n}\right\}$ in a Banach space $\mathcal{Y}$ and a continuous bilinear form $\langle\cdot, \cdot \cdot\rangle$ on $\mathfrak{6} \times$ Y, it holds true that (see e.g. [13])

$$
\lim _{n \rightarrow \infty}\left\langle x_{n}, y_{n}\right\rangle \rightarrow\langle x, y\rangle \text { if } x_{n} \rightarrow x \text { weakly and } y_{n} \rightarrow y \text { weakly. }
$$


In the sequel, we sometimes use the notation $y(x)$ for $\langle x, y\rangle$.

In Theorem 2.2(ii), if we assume $g$ to be bounded, it can be shown that the sequence $\left\{y_{n}\right\}$ obtained from $\left\{\mu_{n}\right\}$ by $y_{n}(A):=\int_{A} g d \mu_{n}, A \in \mathfrak{B}$, converges in Y: $=M(X)$ to $y(A):=\int_{A} g d \mu$ in the $\sigma\left(\mathcal{Y}, \mathcal{Y}^{*}\right)$ topology (that is, the weak topology). On the other hand, assuming without loss of generality, that $g>0$, from Lebesgue's Dominated Convergence Theorem, the bounded sequence $\left\{x_{n}\right\}:=$ $\left\{f_{n} g^{-1}\right\}$ in $\mathscr{G}:=B(X)$, converges to $x=f g^{-1}$ in the $\sigma(\mathscr{G}, \mathcal{Y})$ topology, but not necessarily in the (stronger) $\sigma\left(\mathscr{G}, \mathfrak{G}^{*}\right)$ topology (that is, the weak topology). However, we still have $\lim _{n}\left\langle x_{n}, y_{n}\right\rangle \rightarrow\langle x, y\rangle$, but again, this is because the sequence $\left\{y_{n}\right\} \subset B(X)^{*}$ is in fact in $M(X)$, a special subspace of $B(X)^{*}$, so that the weak convergence of $x_{n}$ to $x$ is not needed.

In fact, the Generalized Dominated Theorem 2.2(ii) can be viewed as the following variant of the Dunford-Pettis property. We recall that for a vector lattice $\mathfrak{6}$, a positive linear functional $y$ is said to be $\sigma$-order continuous if (see Definition 21.5 in [15])

$$
y\left(x_{n}\right) \downarrow 0 \text { whenever } x_{n} \downarrow 0
$$

with $\left\{x_{n}\right\} \subset 96$ and where $x_{n} \downarrow 0$ means that the infimum of the monotone sequence $\left\{x_{n}\right\}$ is 0 . It also implies that $y\left(x_{n}\right) \rightarrow y(x)$ for every sequence $\left\{x_{n}\right\}$ that is orderconvergent to $x$, that is, such that $\left|x_{n}-x\right| \leq w_{n}$ for all $n$ and for some sequence $w_{n} \downarrow 0$ in 96 . This suggests the following:

Proposition 3.1: Let $(96$, Y) be a dual pair of normed vector lattices, with 96 being $\sigma$-complete. Consider two sequences $\left\{x_{n}\right\} \subset \mathfrak{G}$ and $\left\{y_{n}\right\} \subset \mathcal{Y}$, and assume that

(a) $x_{n}$ order-converges to $x$ as $n \rightarrow \infty$.

(b) $\quad 0 \leq y_{n} \rightarrow y$ in the weak topology $\sigma(\mathcal{Y}, \mathscr{O})$.

(c) $y$ is $\sigma$-order continuous.

Then

$$
\lim _{n \rightarrow \infty} y_{n}\left(x_{n}\right)=y(x)
$$

Proof: $\mathscr{S}$ being $\sigma$-complete, if $x_{n}$ order-converges to $x$, then $\inf _{m}>{ }_{n} x_{m}$ is welldefined in $\mathscr{6}$, and so is the "order-liminf" which by definition is $x=$ order$\liminf x_{n}:=\sup _{n}\left[\inf _{m \geq n}\right]$. Similarly, $\sup _{m \geq n} x_{m}$ is well-defined in $\mathscr{S}$ and so is $x=$ order-limsup $x_{n} x_{n}:=\inf _{n}\left[\sup _{m} \geq n_{m}\right]$.

We have in fact, $\inf _{m} \geq n^{x}=: z_{n} \uparrow x$ and $\sup _{m} \geq n^{x} x_{m}=: u_{n} \downarrow x$ as $n \rightarrow \infty$, where $\uparrow$ and $\downarrow$ denote monotone nondecreasing and nonincreasing order-convergence.

As $y_{n} \geq 0$, we have

$$
y_{n}\left(x_{n}\right) \geq y_{n}\left(\inf _{m \geq n_{0}}\left[x_{m}\right]\right) \forall n \geq n_{0}
$$

Therefore, using the convergence of $y_{n}$ to $y$,

$$
\begin{gathered}
\liminf _{n \rightarrow \infty} y_{n}\left(x_{n}\right) \geq \lim _{n \rightarrow \infty} y_{n}\left(\inf _{m \geq n_{0}}\left[x_{m}\right]\right) \forall n_{0} \\
=y\left(\inf _{m \geq n_{0}}\left[x_{m}\right]\right)=: y\left(z_{n_{0}}\right) \forall n_{0} .
\end{gathered}
$$

Now, from the order-convergence of $x_{n}$ to $x,\left\{z_{n_{0}}\right\}$ order-converges to $x$ as $n_{0} \rightarrow \infty$ so that, using $(c)$, 


$$
\liminf _{n \rightarrow \infty} y_{n}\left(x_{n}\right) \geq \lim _{n_{0} \rightarrow \infty} y\left(z_{n_{0}}\right)=y(x) .
$$

A similar argument yields $\lim \sup _{n} y_{n}\left(x_{n}\right) \leq y(x)$ and the result follows.

Examples of Banach lattices $\mathscr{6}$, with property $(3.1)$ are the $L_{p}$ spaces $1 \leq p<\infty$ (see e.g. [15]).

For an $A M$-space like $\mathfrak{G}:=B(X)$, note that every finite measure $\mu$ on $\mathscr{B}$ (hence $\mu \in B(X)^{*}$ ) is $\sigma$-order continuous (cf. [16]), whereas for $X:=[0,1]$ and $9:=C[0,1]$, the Banach lattice of real-valued continuous functions on $[0,1], \mu$ is not $\sigma$-order continuous (see e.g. [15, p. 149]). However, even in the latter case, the following weaker property is still sufficient for Proposition 3.1 to hold,

$$
\int f_{k} d \mu \downarrow 0 \text { whenever } f_{k}(x) \downarrow 0, x \in[0,1],
$$

(by the standard Monotone Convergence Theorem, since $f_{1} \in C([0,1])$ and $\mu$ is finite).

We now see how Theorem 2.2(ii) can be deduced from Proposition 3.1 with an appropriate choice of the spaces $\mathscr{S}$ and $\mathcal{Y}$.

For instance, if the functions $f, f_{n}$ and the measures $\mu, \mu_{n}$ are norm-bounded, choose $\mathfrak{S}_{:}:=B(X)$ and $\mathrm{Y}:=M(X)$, the Banach lattice of finite signed measures on ঔ. Let $\bar{f}_{n}:=\sup _{m}>{ }_{n} f_{n}$ and $\underline{f}_{n}:=\inf _{m}>{ }_{n} f_{n}$ for every $n=1,2, \ldots$. The pointwise convergence $f_{n} \rightarrow f$ implies $\bar{f}_{n} \downarrow f$ and $f_{n} \uparrow \bar{f}$, i.e., the monotone order-convergence of $\bar{f}_{n}$ and $\underline{f}_{n}$ to $f$. Thus $(a)$ in Proposition 3.1 holds for the sequence $\left\{\bar{f}_{n}\right\}$ and $\left\{\underline{f}_{n}\right\}$. The setwise convergence $\mu_{n} \rightarrow \mu$ is precisely the $\sigma(\mathcal{Y}, \mathscr{G})$-convergence, so that (b) also holds. Finally, $(c)$ is satisfied because every $\mu$ is $\sigma$-order continuous. From $\underline{f}_{n} \leq f_{n} \leq \bar{f}_{n}$ for all $n$, it suffices to apply Proposition 3.1 to both sequences $\left\{\bar{f}_{n}\right\}$ and $\left\{\underline{f}_{n}\right\}$ to prove Theorem $2.2(i i)$.

\section{References}

[1] Doob, J.L., Measure Theory, Springer-Verlag, New York 1994.

[2] Dunford, N. and Schwartz, J., Linear Operators, Interscience, New York 1958.

[3] Hernández-Lerma, O. and Lasserre, J.B., Policy iteration in Borel spaces, Acta Appl. Math. 47 (1997), 125-154.

[4] Hernández-Lerma, O. and Lasserre, J.B., Discrete-Time Markov Control Processes: Basic Optimality Criteria, Springer-Verlag, New York 1996.

[5] Hernández-Lerma, O. and Lasserre, J.B., Further Topics on Discrete-Time Markov Control Processes, Springer-Verlag, New York 1999.

[6] Hernández-Lerma, O. and Lasserre, J.B., Fixed points for Markov operators and Markov processes, Proc. London Math. Soc. 76:3 (1998), 711-736.

[7] González-Hernández, J. and Hernández-Lerma, O., Envelopes of sets of measures, tightness, and Markov control processes, Appl. Math. Optim. (to appear).

[8] Langen, H.J., Convergence of dynamic programming models, Math. Oper. Res. 6 (1981), 493-512.

[9] Lasota, A. and Mackey, M.C., Chaos, Fractals and Noise: Stochastic Aspects of Dynamics, Springer-Verlag, New York 1994.

[10] Lasserre, J.B., Invariant probabilities for Markov chains on a metric space, Stat. E Prob. Lett. 34 (1997), 259-265. 
[11] Royden, H.L., Real Analysis, MacMillan, London 1968.

[12] Serfozo, R., Convergence of Lebesgue integrals with varying measures, Sankhya: The Indian J. of Stats. 44 (1982), 380-402.

[13] Schaefer, H.H., Banach Lattices and Positive Operators, Springer-Verlag, Berlin 1974.

[14] Yosida, K., Functional Analysis, sixth ed., Springer-Verlag, Berlin 1980.

[15] Zaanen, A.C., Introduction to Operator Theory in Riesz Spaces, SpringerVerlag, Berlin 1997.

[16] Zhang, X.-D., On weak compactness in space of measures, J. Funct. Anal. 143 (1997), 1-9. 


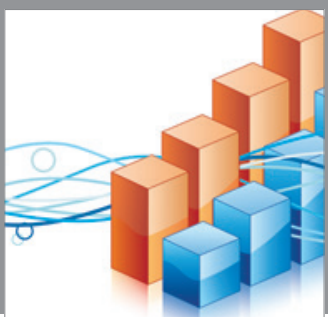

Advances in

Operations Research

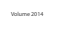

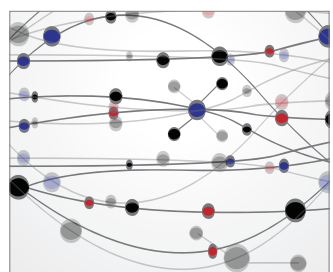

\section{The Scientific} World Journal
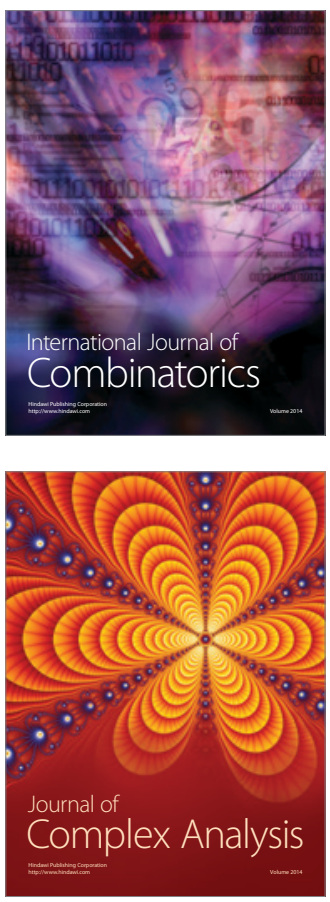

International Journal of

Mathematics and

Mathematical

Sciences
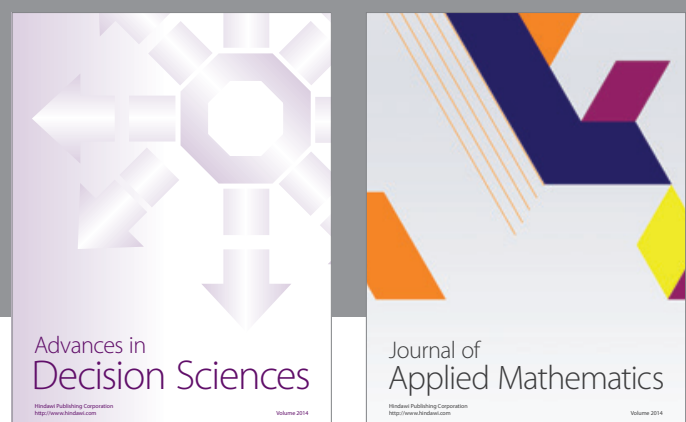

Journal of

Applied Mathematics
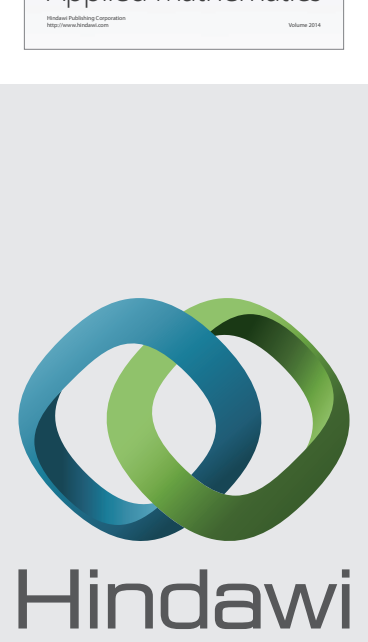

Submit your manuscripts at http://www.hindawi.com
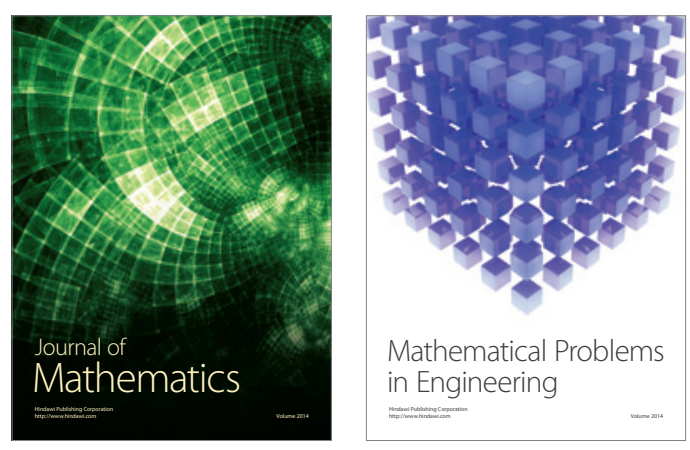

Mathematical Problems in Engineering
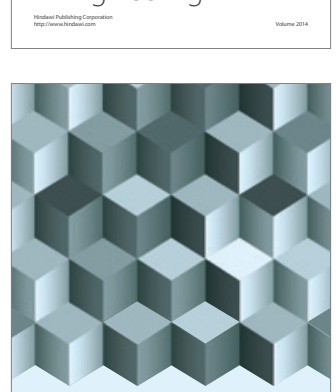

Journal of

Function Spaces
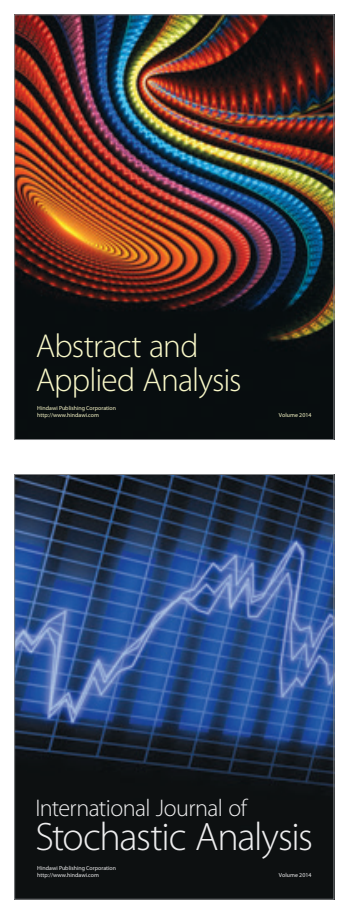

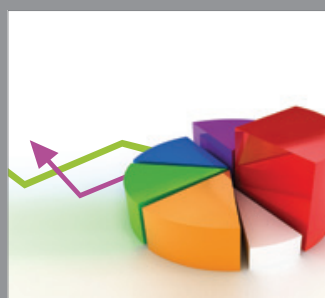

ournal of

Probability and Statistics

Promensencen
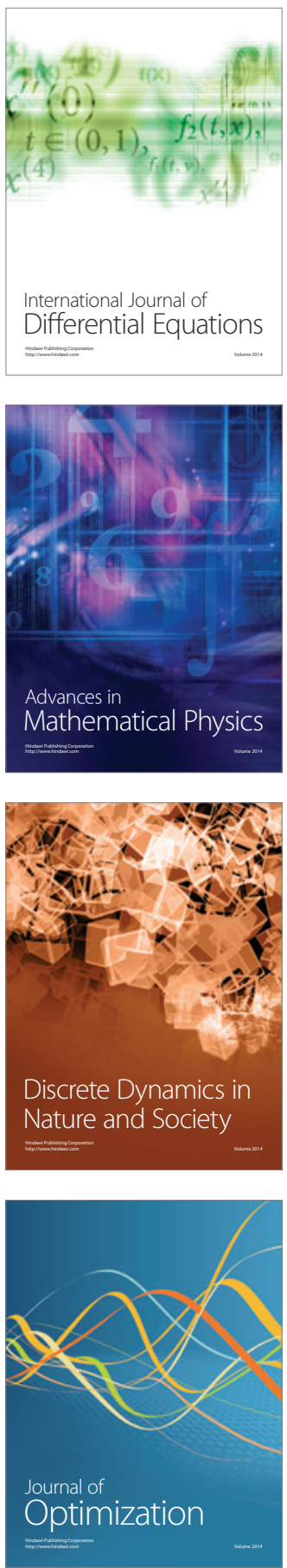